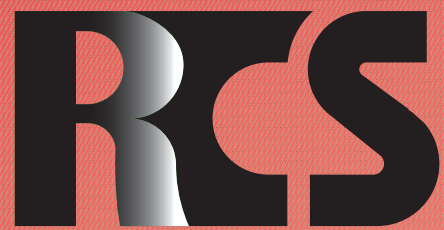

Depósito legal ppi $201502 Z U 4662$

Esta publicación científica en formato digital es continuidad de la revista impresa Depósito Legal: pp $197402 Z$ Z789

- ISSN: 1315-9518 • ISSN-E: 2477-9431

Universidad del Zulia. Revista de la Facultad de Ciencias Económicas y Sociales Vol. XXVI. No.1 


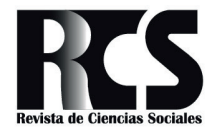

Revista de Ciencias Sociales (RCS). FCES - LUZ

Vol. XXVII, No. 1, 2021, pp. 8-10

- ISSN: 1315-9518 IISSN-E: 2477-9431

\section{Editorial \\ 2020, un año de dificultades y retos tras el COVID-19...}

Un año en el que se vivenció una crisis mundial, de naturaleza distinta a las enfrentadas históricamente (1930,1991, y 2009), cuya causa se ubica en la esfera sanitaria, colocando a los individuos al borde de la existencia. Esta crisis ha tenido efectos severos en el ámbito económico y social, decrecimiento a nivel mundial, la caída del producto interno bruto ha sido abismal, y con ella el desempleo ha sido galopante, haciendo más difícil la supervivencia y el bienestar social de la población mundial. El COVID-19, además de lo mencionado, trajo consigo una reconfiguración en la esfera laboral y educativa, el teletrabajo y la educación remota mediada por tecnología, han sido uno de los retos que ha tocado enfrentar; sin embargo, el más fuerte lo representa el manejo de la salud y estabilidad emocional, puesto en jaque por tantos cambios suscitados, las limitaciones impuestas, el confinamiento, además del temor al contagio.

La crisis sanitaria vivenciada desde finales de 2019, presentada en una magnitud incalculable dado sus consecuencias, observa con esperanza la inmunización a través de la vacunación iniciada en muchos países. El estudio de esta crisis, se ha develado como un fenómeno interdisciplinar y transdisciplinar, donde la ciencia ha venido abriendo soluciones a la misma, no sólo desde la medicina con la vacuna como respuesta; la psicología proveyendo prácticas e intervenciones para fortalecer al individuo como ser emocional, sino también desde la economía, sociología y el resto de disciplinas que buscan contribuciones para enfrentarla.

Los aprendizajes han sido importantes, ha generado reconfiguración, reingeniería a todo nivel, destacando de manera superlativa la del individuo como ser social. Asimismo, en el plano académico, las publicaciones científicas han jugado un rol fundamental, como medios para la difusión y/o socialización de los resultados de la investigaciones, entre estas la Revista de Ciencias Sociales (RCS), con esfuerzo inigualable se mantiene con altos estándares de calidad, cumpliendo con la periodicidad exigidas en revistas, que como ella se encuentran indexadas en importantes índices.

Hoy, en el trasegar a través de la pandemia por COVID - 19, presenta el volumen XXVII, número 1 de 2021, en el que se publican veintisiete (27) artículos, distribuidos entre las áreas administración y gerencia (13), social (6), educación (4), política (2), económica - fiscal (1) y geopolítica (1); desarrollados por investigadores de diferentes ámbitos geográficos como España, Brasil, México, Ecuador, Perú, Cuba, Colombia y Venezuela. Asimismo, se comparten disertaciones relacionadas con la pandemia en Notas de actualidad. Este número de la RCS recrea y enriquece el conocimiento en temáticas tan importantes como las presentadas por área a continuación:

\section{I) Administración y Gerencia:}

1. "Administración de operaciones y su impacto en el desempeño de las empresas", de la autoría de Montejano García, Salomón; López-Torres, Gabriela Citlalli; Pérez Ramos, Marcelo 
de Jesús; y Campos García, Rocío Montserrat. 2. "Gestión de ganancias en el riesgo de quiebra de las empresas públicas mexicanas", cuyos autores son Cumpean Luna, Joel Alejandro; Briseño García, Arturo; y Arango Herrera, Eduardo. 3. "Gestión por procesos en redes de cooperación intersectoriales en la Península de Paraguaná, Venezuela", de los autores Marín-González, Freddy; y Pérez-González, Judith. 4. "Tecnología de información: ¿Herramienta potenciadora para gestionar el capital intelectual?”, del autor Briñez Rincón, Moisés Eduardo. 5. "Turismo comunitario en Ecuador: Apuntes en tiempos de pandemia”, cuya autoría corresponde a Loor Bravo, Lucía: Plaza Macías, Nila; y Medina Valdés, Zoe .

Igualmente en esta misma área se presentan los artículos: 6. "Turismo minero en Yanacocha: Una alternativa de desarrollo para la región de Cajamarca-Perú”, de los autores Nugra Betancourth, Mónica Alexandra); Illescas Espinoza, Wilmer Henry); Cuadros García, Paquita Alejandra; y Valdivia Ramos, Roman Arturo. 7. "Condiciones de mercado para la demanda nacional del Sacha Inchi en Ecuador”, presentado por · Preciado Ramírez, Joffre Danny; Alcívar Soria, Evelyn Eugenia; Prado Chinga, Alberto; y Guerra Herrera, Kleber Santos. 8. "Gestión y costos de producción: Balances y perspectivas", de Casanova Villalba, César Iván; Núñez Liberio, Rosa Verónica; Navarrete Zambrano, Cecilia Mercedes y Proaño González, Esther Angélica. 9. "Emprendimiento e innovación: Dimensiones para el estudio de las MiPymes de Azogues-Ecuador”, en el que figuran como autores Solis Muñoz, Juan Bautista; Neira Neira, Mercedes Lucia; Ormaza Andrade, Jorge Edwin; y Quevedo Vázquez, Jorge Oswaldo. 10. "Gestión administrativa de las instituciones de educación superior: Universidad Católica de Cuenca-Ecuador”, de la autoría de Ordóñez Parra, Janice; Cárdenas Muñoz, Jorge; Cuadrado Sánchez, Gina; y Zamora Zamora, Geovanny. 11. "Revalorización y sostenibilidad tecnológica de los andenes interandinos agroecológicos en la región Arequipa-Perú", cuyos autores son León de los Santos, Nelva; Pérez Postigo, Gerber Sergio; Becerra Castillo, Sócrates; y Rodríguez Quispe, Jorge Louis. 12. "Marketing y la demanda de viviendas sostenibles en Perú”, presentado por Alvarez Luján, Blanca Lina; y Zulueta Cueva, Carlos Eduardo. 13. "Universidad autónoma venezolana: Perspectiva gerencial de una organización social", de los autores Caira Tovar, Norma M.; Sánchez Morles, José G.: y Lescher Soto, Isaías S.

\section{II) Social:}

1. "COVID-19 y comunidad gitana: Enfoques en la prensa española", cuyos autores son Muyor Rodríguez, Jesús; y Segura Sánchez, Antonio Jesús. 2. "Personas jóvenes con discapacidad y el papel que juega la familia en su ocio", de los profesores Madariaga, Aurora; Romero, Sheila; Romera, Liana; y Lazcano, Idurre. 3. "Combate a la pobreza y percepciones de beneficiarios en la frontera sur de México”, presentado por los profesores Sorzano Rodríguez, Deisy Milena; Rocha Romero, David; y Acosta Torres, Armando. 4. "Participación ciudadana en el sistema de seguridad social en salud en Colombia”, cuyos autores son Restrepo Pimienta, Jorge Luis; Cotrina Gulfo, Yamid Enrique; y Daza Suarez, Alfredo. 5. "Publicidad y construcción de un imaginario social: Representación del género femenino en televisión ecuatoriana”, de los profesores Ramírez, Alicia Elizundia; y Álvarez Yaulema, Myriam. 6. "Transgénero: Un análisis desde la mirada de los derechos humanos", de la autoría de Cedeño Barreto, Mercedes de Los Ángeles.

\section{III) Educación:}

1. "Doctrina económica-financiera y contable: Un reto en la educación infantil", presentado por Guevara Garzón, Catherine Ninoska; y Rodríguez Bolívar, Liliana Margoth. 2. "Formación del emprendimiento social: Compromiso de la Universidad de la Guajira en Colombia", de los autores Cantillo Campo, Nair; Pedraza Reyes, Carlos Julio; y Suarez Barros, Horacio. 3. "Nuevas tecnologías en los primeros subniveles de Educación Cultural y Artística 
en Ecuador", presentado por los profesores Crespo-Fajardo, José Luis; y Pillacela-Chin, Luisa. 4. "Comportamiento ecológico y cultura ambiental, fomentada mediante la educación virtual en estudiantes de Lima-Perú", de la autoría de Yangali Vicente, Judith Soledad; Vásquez Tomás, Melba Rita; Huaita Acha, Delsi Mariela; y Baldeón De La Cruz, Maruja Dionisia

\section{IV) Política:}

1. "Nueva propuesta de financiamiento público a partidos políticos en México", de los profesores Arredondo, Verónica; Vega Esparza, Reina Margarita; Villegas Santillán, María Teresa; y Álvarez Diez, Rubén Carlos. 2. "Ciberactivismo: Elemento articulador del Movimiento Indigena del Cauca, Colombia”, de la autoría de García-Villalba, Cristian; y Gutiérrez Zamudio, Mayra Alejandra.

\section{V) Económica - Fiscal:}

1. "Impacto de las transferencias federales en la deuda municipal de los ayuntamientos mexicanos”, de los profesores Herrera Díaz de León, Luis Lenin; González Acolt, Roberto; Alcantar López, Cristian Omar; y Govea Franco, Ángeles Montserrat.

\section{VI) Geopolítica:}

1. "Revisión contextual para la interpretación del análisis de riesgo geopolítico”, de los autores Baena-Rojas, José Jaime; y Bonilla-Calle, Daniel

Este conjunto de artículos, que a través del volumen XXVII, número 1, de la Revista de Ciencias Sociales (RCS) se colocan a disposición de la comunidad científica, son demostrativo del compromiso con la academia y la mística por continuar, a pesar de la vicisitudes, en el camino de socializar los resultados de investigaciones.

Dra. Lissette Hernández - Fernández

Ex Editora - Jefe de la Revista de Ciencias Sociales (RCS) Profesora Tiempo Completo. Universidad del Atlántico (Colombia) Profesora Emérita. Universidad del Zulia (Venezuela) E-mail: lisettehernandez@mail.uniatlantico.edu.co ORCID: https://orcid.org/0000-0003-0839-6057 